\title{
Constructing Routes of New Software Development Method
}

\author{
Yu Gao \\ Computer department,, Zhejiang Ocean University,, Zhoushan, Zhejiang, P.R.China \\ gaoyu@zjou.edu.cn
}

\begin{abstract}
As the rapid development of computer, the quondam software development methods may not meet the requirements, people need constructing new software development methods. In order to construct new software development method, it is necessary to research the "constructing routes of new software development method". For existing software development methods, we carry out a comprehensive, detailed analysis and summary. On this basis, the four constructing routes have been proposed. These constructing routes are: The first constructing route is "to propose new development thought". The second constructing route is "to improve defects of the existing software development methods". The third constructing route is "integrating the advantages of certain software development method". The fourth constructing route is "with the help of new software development tools".
\end{abstract}

Keywords - Software engineering; Software development method; Constructing route

\section{INTRODUCTION}

Software development method is one of the important part of software engineering theory.

Before 1970, the software crisis spread. Analyzing reasons of software crises, we find that: "Without the right software development method is one of the key reasons of producing the software crisis".

In order to remove the software crisis, to improve the efficiency and quality of software development, people began to research software development method. In 1970, the famous waterfall model emerged. In early 1970's, structured programming methods formed, in its basis, structured software development method gradually formed. In early 1980's, at object-oriented languages (Smalltalk and $\mathrm{C}++$ as representative) basis, object-oriented software development method developed gradually. Later, rapid prototyping model, incremental model and spiral model successively emerged. Later, some formal software development methods [1] and component-based software development methods [2] successively emerged. To 1990's, some Agent-Oriented software development methods successively been proposed, for example MaSE method, Gaia method, Tropos method and so on [3][4]. To late 1990's, based on the UML (Unified Modeling Language), RUP method [5] been proposed. Afterwards, some Agile software development methods [6][7] ( including XP) appeared. In recent years, with the popularity of the Internet, based on SOA (Service-Oriented Architecture) software development methodology [8] appeared. The wide use of software development method effectively alleviated the software crisis, and improved efficiency and quality of software development.

Analyzing the existing software development methods, we can see that: "To every a software development method, it is created through research and practice, the purpose is solving a particular problem, in the particular case.". Therefore, a software development method is suitable in a particular area, solving a particular problem. With the development of the computer, there will be new areas of computer applications, new circumstances and new problems may appear. New circumstances and new problems may also appear in existing computer applications field. The face of new areas, new circumstances and new problems, the existing software development methods may not suit, it may be difficult to complete a new software development tasks. Therefore, people need constructing new software development method.

In order to construct new software development method, it is very necessary to research the "constructing routes of new software development method". That is to say, people should research the "How to construct new software development method?". In the past, although people successively constructed some software development methods, but the research of "constructing routes of new software development method" has not attracted people's attention. Currently, no one specializes in "constructing routes of new software development method".

We deep researched "constructing routes of new software development method". After the research, we found the four constructing routes of new software development method. These constructing routes are: The first constructing route is "to propose new development thought.". The second constructing route is "to improve defects of the existing software development methods". The third constructing route is "integrating the advantages of certain software development methods". The fourth constructing route is "with the help of new software development tools".

In the following, we elaborate on the research content.

\section{CONSTRUCTING ROUTES OF NEW SOFTWARE DEVELOPMENT METHOD}

\section{A. To Propose New Development Thought.}

We investigate some important software development method, and analyze their development thought.

Many software development methods used development process of linear sequence. They are in accordance with the software life cycle, and the development process is divided into several small stages. When the development, a stage by one stage in order of development, the each stage carries out a specific task. Waterfall model is representative of such software development methods. Such software development methods reflected a linear development thought. Linear development thought is simple, and linear development thought emphasizes stage and sequence. The software 
development method of following the linear development thought is suitable for the clear user needs, and is suitable for the development of less complex tasks.

Many software development methods used development process of repeated cycle. Rapid prototyping model is representative of such software development methods. In constructing prototype system, the rapid prototyping model uses repeated cyclic manner, each prototype than the last prototype closer to the real needs of users. When customer feel satisfied, the cycle of constructing prototypes stop. Such software development methods reflected an iterative development thought. Iterative development fit law that people recognized things, it uses the way of repeatedly recognized and practice, it can gradually deepen understanding things. For iterative development, it requires several iterations process, each iteration process has a new harvest, things to be understanding gradually deepening and integrity. For the circumstances that user requirements are not entirely clear and user requirements are to changing, iterative development thought is suitable.

Emergence of the concept of classes and objects reflected an unprecedented development thought. The core of this new development thought is put the data and method of operation data together. Under the guidance of the new development thought, object-oriented software development methods grow up. Compared with the traditional development thought, when people carry out software development, the new development thought has very obvious advantages. Advantages include: between various stages (system analysis, system design and system implementation), the transition is the very nature; software maintainability has a very large increase. Now, object-oriented software development methods have become the mainstream development method.

In the development software, many software development methods reuse various software components. To be reuse the software component can be a program module code, and can be other software components (such as design program, test program, etc.). Based on COTS software development method [9] or based on component software development method is representative of such software development methods. Following this development thought by such software development methods is "reiteration use". The application of this development thought can save development time, and speed development process, software quality is greatly improved. Now, the software development methods of following this development thought are applied very widely.

Agile methods are joint name of a class software development methods, XP (eXtreme Programming) is a typical representative of Agile methods. In 2001, Agile Alliance published Agile Manifesto, and announced their new values to the world: (1) emphasis on individual creativity and communicate with each other more important than the software process and software tools, (2) emphasis on the current software more important than understanding the document, (3) emphasis on user cooperation more important than solely rely on the contract, (4) emphasis on responding of change more important than following the plan only. Agile Manifesto reflects a new development thought, and the new development thought is root causes of generating variety Agile software development methods. Variety Agile methods follow new development thought, to such phenomena that the traditional development process is complex and cumbersome, and a large number of documents are produce and maintain, and timely response to changes is lacking, it emphasizes human-centered, adapting change, simplicity and rapid feedback, it makes the development efficiency to enhance greatly. Agile methods applied to complex fickle situations, and applied to medium-scale or small-scale development projects.

In the above, we researched some existing software development methods, we observed that: according to different development thought, different software development method is constructed. Under the guidance of different development thought, they shown different form respectively, they have different characteristics respectively, they were applied to different areas respectively, they can solve different problem respectively.

As described above, any software development methods must be constructed by following some kind of development thought. Therefore, we found a "constructing route of new software development method". Constructing route is that "People can put forward a new development thought. A new software development method is construct, which is based on the new development thought."

For example, SOA (Service-Oriented Architecture) to appear in recent years is a good example. Traditional software development thought require that software relies on the same technology or platform, and require that software components more closely linked, and require that software can not be distributed in different physical locations. New development thought based on SOA is that the new system relies on a network, and the new system is constituted by application software at different physical location and based on different technology or platform. The basic features of the new system are loosely coupled and coarse-grained, the various parts of the new system linked together by mutual services. Under the guidance of the development thought, people put forwarded some SOA-based software development method [8][10][11].

In fact, if people can put forward the new development thought, then, according to the new development thought, the development method of constructing must be new software development method, and general they are important software development methods. This can be seen from the above description.

\section{B. To Improve Defects of the Existing Software Development Methods}

We investigate some software development method, and analyzing the case that they appear by to improve defects of the existing software development methods.

In the early days, when doing analysis and design of software systems, all software development methods are based on natural language. Since the using natural language is simple and convenient, in doing analysis and design. However, because natural language itself is not rigorous, in describing the software system, the contradictions, vague, incomplete and other phenomena may arise. This is a common defect of various software development methods in the early days. This defect may lead to software quality problems. To the defect, when 
people search countermeasures, mathematical language has been thought of. Mathematical language is clear, rigorous, no-contradictory. If we borrow mathematical language to describe the software system, the contradictions, vague, incomplete and other phenomena can be avoided. So at software development process, people introduce mathematical language, using mathematical language analyze and design software system. In this way, formal software development method appeared. This is a new software development method that is different from traditional software development methods. The emergence of formal software development method has opened up a path for to ensure software quality and to enhance software reliability. But its application is also subject to some restrictions, such as when software is every large and very complex, using mathematical language to describe the user's requirements is very difficult.

To the traditional software development methods, after delivery of the software system, the change of software is very difficult. But, if the external environment changed, or the user's requirements changed, this will force software changes. This will give developers to increase a lot of work, and the user is not convenient. If a flexible software system can be design, in a certain range, it can meet and adapt to the changing environment and user needs, then, the workload can reduce, user is also convenient. However, traditional software development methods can not do this. To the defect of traditional software development methods, people put forward the concept of flexible software, and put forward flexible software development methods [12], traditional software development methods are improved. At present flexible software and flexible software development method still is in the research. Flexible software can be divided into flexibility of facing the general user and facing the developers, component technology can be use in developing flexible software [13].

Most software development methods of component-based look at the static assembly of component. That is to say, the selection and assembly method of component are determined in design phase. Common defects of such software development method is the lack of flexibility, the system's adaptive capacity is weak. To defect of such software development method, the literature [14] proposed a new software development method. The new software development method improved traditional software development method of based on the component, a role-based dynamic component assembly model is proposed. This model make that application system have more flexible at to select and to assemble components, system's adaptability can increases, the system's maintenance costs can reduces.

Incremental model is a software development method widely to be used. Concurrent incremental model is a software development method of greater risk than the basic incremental model. Although the concurrent incremental model has advantages, but, because analysis and design of each incremental is concurrent, therefore, the concurrent incremental model may lead to no suitable each other for every increment, it must be constantly repaired. At the same time, due to concurrent incremental model is only simply to pursue shortening the development cycle and reducing cost, it will have a negative impact on software quality. To defect of concurrent incremental model, [15] proposed a new software development method. At basis of concurrent incremental model, people carry out improving. People proposed a three-stage parallel pipeline software process model. This model can improve software quality and productivity, and can reduce cost and risk.

In the above, by investigating we observed that: "Since the old software development method have defects, after improving the defects, a new software development method has been constructed."

Since in the past people can follow this route, then in the future people certainly can construct new software development method according to this route. Such, we found a "constructing route of new software development method". Constructing route is that "To defects of old software development method, after improving the defects, the new software development method is constructed."

In fact, using this route, to be constructed the new software development method greatly can expand scope of old method, and can enhance development capabilities of old method, and can extend the use cycles of old method.

\section{Integrating the Advantages of Certain Software Development Methods}

We investigate some software development method, analyzing case that they integrating the advantages of certain software development methods.

We analyze a software development method to be proposed in [16]. This software development method integrated two methods together. One is object-oriented method of UML -based. Another one is formal method. To the object-oriented method of UML-based, UML provides variety graphics, such as class diagram, collaboration diagram, component diagram, use case diagram, activity diagram, deployment diagram, and so on. When developers analyze and design software systems using these graphical, it is both intuitive and convenient, but to eliminate ambiguity is difficult. Tightness, reliability and verifiability of formal software development method are very good, but the software development process is not intuitive, and does not meet the developer's habit. Above two methods have respective advantages and disadvantages, the advantages of each method exactly make up for shortcomings of another method. If to integrate advantages of above two methods, constructing a new software development method, then result apparently is better than using one method. In [16], the author is based on this fact, and the advantages of both methods are integrated, to form a new software development method.

Literature [17] proposed a software development method. This software development method has integrates object-oriented method, prototyping method and CASE method together. This software development method divide into relatively independent some stage for all development activities. According to function of system, the system was divided into some subsystem. Each subsystem is relatively independent. Complexity of each subsystem is relatively low. At all stages of the development process, and in the process of developing various subsystems, according to the situation, object-oriented method or prototype methods or CASE method were used. Because object-oriented methods, prototyping methods and CASE methods each have their 
own advantages and disadvantages. Now to integrate them together, give play to their respective advantages, this obviously is better than using a certain method. In [17], first, content of new software development methods been introduced, and then combined with development work of large-scale MIS (management information system), using case of the new software development methods been introduced.

We see [18], an incremental and iterative software development method has been proposed. This software development method integrates incremental model, waterfall model and rapid prototyping model together. When using this software development method, the software system is divided into several increments. For each increment, waterfall model is used to guide development. For the entire system, method of constructing prototype is used, to carry out development iterative. In the each iteration, a prototype was obtained. Development tasks complete through a number of iterative. One of the most important features of rapid prototyping model is iteration. This feature makes it to have very good ability to adapt to change. Incremental model suited to the environment constantly changing to user needs and conditions, incremental model is beneficial to reduce the development risks. A new software development method in literature [18] to be proposed, integrate the advantages of several development methods. Software development method of constructing as such, the ability to adapt changing is better.

By analyzing the above examples, we observed that: "In the past, through approach of integrating advantage, some new software development methods were constructed."

Such, we found a "constructing route of new software development method". It is that "According to the needs of solving question, we can integrate the advantage of several software development methods, on this basis, constructing new software development method."

In fact, after 1990's, many software development methods appear according to this constructing route. Some software development methods are simply integrating the advantages of several software development methods. For example, the situation was described, in [16], [17] and [18]. Some software development methods also added some other content, after integrating the advantage of several software development methods. For example, front talked about the XP, XP is a typical representative of Agile methods, it not only integrates the advantages of several software development methods (such as the prototype model, incremental model), but also added its own unique "Agile" development thought (emphasizing the four core values: communication, simplicity, feedback, courage).

\section{With the Help of New Software Development Tools}

Let's observe some the software development tools, and analyze relationship of these tools and software development method.

Data flow diagram is an important software development tool, and its emergence is relatively early. It is also well-known tool. In various kinds traditional structured software development methods, data flow diagram is used for system analysis and design [19[[20]. Data flow diagram is one of the important elements of the software development method. Or other, formation of some software development is by means of data flow diagram. When doing system analysis and system design, data flow diagram is the center of software development method. If there is no data flow graph, then these software development methods does not appear.

Now, UML (Unified Modeling Language) is very common software development tools [21]. By means of UML, people constructed some new software development method, and RUP is one of the most famous. RUP adopt the iterative development, and adopt visual modeling and to reuse components. In RUP, the architecture is as a central, and product is build and test incremental. RUP need to set up a variety models for systems analysis and design. Model needs the help of UML. UML provides variety graphics. These graphics have played a very important role in RUP, such as use case diagrams, collaboration diagrams, component diagrams, activity diagrams, deployment diagrams and so on. There are also some software development methods by means of UML, such as in [22] a software development method is introduced. This software development method construct "use-case model" using "use case diagram", and construct "static structure model" using "class diagram and object diagram”, and construct "dynamic behavioral model" using "sequence diagram, collaboration diagram, state diagram and activity diagram”, and construct "physical structure mode" using "component diagrams and deployment diagrams". To make a smooth transition to the four models, "architecture model" was also constructed. UML was as description language of "architecture model".

Now, Struts is an effective tool for Web developing. Struts provide a framework to build based on MVC (Model-View-Controller) applications. Meanwhile, Struts also provides library and utilities for faster and easier development. Initially, Struts was created by Caig R.Mc Clanahan. In 2000, Struts were used for the Jakarta project of Apache Software Foundation (ASF). In June 2001, Struts1.0 released, since then Struts develop rapidly. With Struts, a new software development method evolved. This new software development method is characterized by: "After systematic analysis and preliminary design to be done, design and implementation of model, view and controller must be carried out." To follow this new software development method, many software systems have been developed. For example, in the literature [23]-[26], the contents are introduced in this regard.

By analyzing the above examples, we observed that: "Software development tools and software development methods are closely related. Many software development methods rely on certain software development tools (and probably rely on many software development tools, but in these tools, only one is most important). As a specific software development methods rely on a specific software development tools, therefore, from the perspective of software development tools, this software development method is different from other software development methods. If specific software development tools do not exist, then this software development method does not exist. If the software development tool has changed, then the content and name of the software development method sure is also changed."

By analyzing the above examples, we found a "constructing route of new software development method". 
It is like this: "If we can construct a new software development tools, then we can construct a new software development method, new software development tools can be used as the center of new software development method."

In fact, people have been looking for better software development tools, and software development tools have been constantly updated, but the pace just is slower. If once emergence of new software development tools, and it can significantly improve efficiency and quality of development, then, people can construct new software development method using new software development tools as the center.

\section{CONCLUSION}

Above we researched "constructing route of new software development method". We particular discussed the four constructing routes of new software development method. To put forward the four routes is based the on detailed analysis and summary to various existing software development methods.

Recalling the history of software development activities, the first route (to propose new development thought) is the most important. According to this route, to be constructed software development methods were important software development methods. Of course, by the route to construct a new software development method is also very difficult.

According to second constructing route (to improve defects of the existing software development methods) and third constructing route (integrating the advantages of certain software development methods), difficulty constructing new software development method is relatively smaller. Therefore, in recent years, according to the two routes, many software development methods were constructed. However, their impact was not significant.

According to fourth constructing route (with the help of new software development tools), constructing software development method is also very difficult. Since, generation of an important software development tool is not an easy task. In order to improve development efficiency and quality, people have been working on new software development tools. We look forward to better software development tool to promote the development of software development methods.

\section{REFERENCES}

[1] Roger S Pressman. Software Engineering: A Practitioner's $\begin{array}{lll}\text { Approach. Sixth Edition } & & \end{array}$ York:McGraw-Hill,2005:802-846

[2] WAN Lin-rui, HU Hong, SUN Hong-xing . Researches on Component Oriented Software Development Methodology [J]. Shenyang: MINI- MICRO SYSTEMS, 2003,24(3):365-370

[3] Gerhard Weib. Agent orientation in software engineering [J].The Knowledge Engineering Review, 2001 ,16 (4):349-373

[4] Ofer Arazy , Carson C Woo. Analysis and design of agent-oriented information systems [J]. The Knowledge Engineering Review , 2002,17 (3): 215-260
[5] Philippe Kruchten.The Rational Unified Process: An Introduction, Third Edition [M].Addison Wesley,2004

[6] SHEN Bei-Jun, CHEN Cheng, JU De-Hua. Research on agile software process [J]. Beijing: JOURNAL OF COMPUTER RESEARCH AND DEVELOPMENT, 2002, 39(11):1456-1463

[7] Highsmith J, Cockburn A. Agile software development : the business of innovation [J]. Computer, 2001,34 (9):120-121

[8] WEI Dong,CHEN Xiao-jiang,FANG DING-yi. Research on the Software Development Based on SOA[J]. Microelectronics \& Computer,2005,22(6):73-76

[9] QU Xi-long. Research for Development of COTS-based Software System [J]. Chengdu:Application Research of Computers,2005,(9):43-45

[10] YE Yu , etc. Research of SOA and Its System Building [J]. Chengdu:Application Research of Computers, 2005,(2),32-34

[11]ZHANG Hai-jun, SHI Wei-feng, LIU Wei. Research and implementation enterprise application integration framework based on SOA [J]. Beijing: Computer Engineering and Design, 2008,29(8): 2085-2088+2092

[12] SHEN Li-min . Flexible Software Development Technique [M] Beijing: National Defence Industry Press, 2003

[13] NIU J ing-chun ,SHEN Li-min ,YANG Hua-lin. Study on Method of Flexible Software Development Based on Component [J]. Xi'an: COMPUTER TECHNOLOGY AND DEVELOPMENT, 2009,19(9):72-75

[14] WANG Jun, FAN Zhi-ping. A Dynamic Assembled Model of Components Based on Role[J]. Chengdu: Computer Applications,2003,23(2):5-8

[15] ZHAO Peng. A three-phase parallel-pipelining software process model [J]. Haerbin: JOURNAL OF NATURAL SCIENCE OF HEILONGJ IANG UN IVERSITY, 2006, 23(2):245-249

[16] MING Zhong, Research on Software Developing Method of Integrating UML and RAISE [J]. Shanghai: Computer Engineering, 2001,27(4):23-25

[17] Li Hongbo,Shao Xiuli,Tu Fengsheng. Application and Research in a Large-scale MIS Development with Method Based on Compound of Object-oriented, Prototype and CASE [J]. Beijing: Computer Engineering and Applications, 2003, (2):119 122

[18] HAN Cheng-shuang. Incremental iterative software development method [J]. Hefei: JOURNAL OF HEFEI UNIVERSITY OF TECHNOLOGY, 2004, 27(6):714-717

[19]Zhang HaiFan, Software Engineering Introduction (Fifth Edition)[M]. Beijing: Tsinghua University Press, 2008:104-112

[20]Zhang Gang,Wang Tongsheng. A New Design Method for Conversion of DFD Into Structured Diagram [J]. Beijing: Journal of Computer Research and Development,1993,(11):55-60

[21] Roger S Pressman. Software Engineering: A Practitioner's Approach. Sixth Edition[M]. New York: McGraw-Hill,2005:94-102

[22] Ma Chongming,Zhang Xuewang,Fan Shikping. The UML-Based Software Architecture Development Methodology [J]. Beijing: Computer Engineering and Applications, 2006,(4):118-120+148

[23] ZHANG Qiao, SUN Xiao-wei. Design and implementation of ERP based on Struts framework [J]. Beijing: Computer Engineering and Design,2007,28(9):2148-2149+2153

[24] ZHU Xi, etc. Application of Struts Framework for Intelligent Building Property Management System [J]. Xi'an: COMPUTER TECHNOLOGY AND DEVELOPMENT, 2006,16(2):62-64

[25] WANG Jian-wen, WANG Min. Research and implementation of information management system of oil field based on struts framework [J]. Beijing: Computer Engineering and Design, 2009,30 (13):3231-3233

[26] YANG Yong, HAN Li-ying. Application of Struts Framework Based on MVC Design in E-commerce System[J]. Chengdu: Application Research of Computers, 2006 (5):172-174 